\title{
La competencia digital de la ciudadanía, una necesidad creciente en una sociedad digitalizada
}

\author{
Digital competence of citizens: a growing need in a digitalized society
}

\author{
(iD) Juan Eusebio Silva Quiroz; juan.silva@usach.cl \\ Universidad de Santiago de Chile (Chile) \\ José Luis Lázaro-Cantabrana; joseluis.lazaro@urv.cat \\ Universitat Rovira i Virgili (España)
}

\section{Resumen}

La sociedad del conocimiento ha generado una nueva alfabetización de carácter digital que, por sus aristas e implicancias en la educación, en la formación y en el ámbito laboral, se conoce con el término de competencia digital. Este artículo aborda la problemática de la competencia digital en la ciudadanía, entendida como un elemento clave para desenvolverse en la sociedad del conocimiento y aprovechar las potencialidades de la tecnología digital y las oportunidades que esta brindan en diferentes ámbitos del desarrollo profesional y personal de las personas. Para tal efecto, se aborda la problemática de la competencia digital, la brecha digital, como elemento que dificulta el desarrollo; y se propone un conjunto de recomendaciones que facilitarían el desarrollo de la competencia digital en la ciudadanía.

Palabras clave: competencia digital, ciudadanía, brecha digital, evaluación

\begin{abstract}
The information society has generated new digital literacy which, because of its aspects and implications for education, training and the workplace, has coined the term digital competence. This articled deals with the problem of digital competence of citizens, understood as a key element for performing in the knowledge society, and putting the potential and opportunities offered by digital technology to its best use in different areas of professional and personal development of individuals. To this end, it addresses the issue of digital competence, the digital divide as an element that hinders development and proposes a set of recommendations that would facilitate the development of digital competence of citizens.
\end{abstract}

Keywords: Digital competence, citizenship, digital divide, assessment 


\section{INTRODUCCIÓN.}

La educación y la formación son un elemento fundamental en el desarrollo de cualquier sociedad. En la Declaración de Roma (Council of the EU, 2017), los estados miembros de la Unión Europea enfatizan la necesidad de formar a los futuros ciudadanos mediante un sistema educativo equitativo, que les permita acceder al trabajo y que se adapte a las necesidades de la era digital (EUCO, 2017).

La European Commission (2018a) elabora el Plan de Acción de Educación Digital, mediante el cual se ofrecen orientaciones para poder realizar un mejor uso de las tecnologías digitales (TD) y así desarrollar la competencia digital (CD) necesaria para el trabajo y para la vida cotidiana de los ciudadanos de una sociedad propia de la era digital. La transformación digital de la actividad económica e industrial producirá efectos importantes en cuanto a las capacidades profesionales que se necesitarán para poder acceder al empleo en los próximos años (European Commission, 2017). Sin duda, la CD jugará un papel importante para poder capacitar a los ciudadanos de manera que les permita adaptarse mejor y más rápido a los cambios producidos por la constante digitalización de la sociedad (European Commission, 2018b).

Del mismo modo, la formación en CD puede producir oportunidades y efectos de desigualdad. Reducir la brecha digital existente entre los ciudadanos y entre los diferentes países debería ser una prioridad para promover el crecimiento inclusivo de una sociedad más justa (Lázaro, Estebanell y Tedesco, 2015). El concepto de brecha digital suele asociarse a la diferencia de acceso a las TD que existe entre los países más desarrollados y los que están en vías de desarrollo (Tello, 2007) o a las inequidades sociales en materia de acceso, uso y apropiación de las TD (De la Selva, 2015). La brecha digital puede considerarse como la nueva desigualdad del siglo XXI. Ahondando un poco más en este término, observamos que, como factores de riesgo, coexisten con los anteriores aspectos relacionados a la conectividad (vinculados a la calidad de la conectividad) y a la comunicabilidad (entendida como el uso libre de las TD) (CEPAL, 2009; Duarte y Pires, 2011). Un concepto más reciente vinculado a la brecha digital es el de participación ciudadana (Fernández, 2017). La posibilidad de participar en los procesos democráticos en línea de la sociedad junto con la participación en la sociedad del conocimiento produce, cada vez más, efectos de desigualdad y una distancia mayor entre aquellas personas que son capaces de multiplicar su capacidad de aprender y adquirir conocimientos. Este efecto de distanciamiento se asocia al concepto de brecha cognitiva (Tello, 2007).

La Agenda 2030 (United Nations, 2015), mediante los Objetivos de Desarrollo Sostenible, en el objetivo número 4 propone garantizar una educación inclusiva, equitativa y de calidad y promover oportunidades de aprendizaje durante toda la vida para todos. Para este propósito resulta necesario promover acciones innovadoras en educación que contemplen la utilización de las TD y el desarrollo de la CD de los estudiantes (European Commission, 2018b). El uso de las TD en situaciones de aprendizaje diversas y en diferentes contextos debería contemplarse como una oportunidad, no como una amenaza. Los contextos de aprendizaje no formal e informal DOI: https://doi.org/10.21556/edutec.2020.73.1743

Página $38 / 50$ 
enriquecen los procesos de educación formal que se desarrollan en el sistema educativo reglado (Cobo y Moravec, 2011). Los aprendices actuales, vinculado al concepto de nativos digitales (Prensky, 2001), utilizan la tecnología de forma natural e intuitiva. Aun así, en el ámbito educativo se pone de manifiesto que los jóvenes actuales precisan de una guía, por parte del docente, en lo que al uso efectivo de las TD para aprender se refiere (Cabezas y Casillas, 2017; Gisbert y Esteve, 2011).

Si bien todo lo expuesto podría relacionarse con el concepto de alfabetización digital, consideramos que debemos vincularlo a una propuesta más amplia, más allá del uso correcto de las diferentes TD. El concepto sería el relacionado con la CD orientada al "desarrollo de las habilidades comunicativas, del sentido crítico, la participación o la capacidad de analizar la información a la que se accede, entre otras" (Fundación Telefónica, 2020). Este proceso toma especial relevancia en la formación universitaria, en la que los estudiantes y necesitan desarrollar, de forma necesaria, su autonomía y su capacidad de aprender utilizando las TD de forma permanente, a lo largo de su vida, para poder adaptarse profesionalmente a los continuos avances de la sociedad digital (Sánchez-Caballé, Gisbert y Esteve, 2020).

En este contexto, la situación generada por la pandemia mundial del COVID19 ha acelerado los procesos de adaptación a la tecnología de la educación, a la vez que se han hecho evidentes los problemas derivados de la digitalización y la necesidad de virtualizar los procesos de enseñanza y aprendizaje. Además de los problemas derivados de las posibilidades de acceso a los dispositivos y a una conectividad de calidad, han aflorado las dificultades en el uso de las TD, producto de la falta de CD. Y, por añadidura, el ámbito educativo, la población ha necesitado utilizar las TD en el ámbito laboral (el teletrabajo ha resultado ser una solución en muchos casos), en el ocio y las relaciones personales y sociales.

Este momento es una buena oportunidad para plantearnos algunas cuestiones como ¿qué nivel de $C D$ poseen los ciudadanos?, ¿cómo podemos medir el ese nivel de CD?, ¿cómo podemos mejorarlo? Este artículo busca situar la problemática de la CD e intenta ofrecer algunas miradas para dar respuesta a estas preguntas. Entendemos que el escenario actual es una gran oportunidad para destacar la CD de la ciudadanía como aspecto clave para el e-aprendizaje, el e-comercio, la eciudadanía, y los diferentes formatos de uso de la TD que apelen a contar con ciudadanos competentes digitalmente.

\section{LA COMPETENCIA DIGITAL}

El ciudadano del siglo XXI debería poseer una serie de conocimientos, habilidades y actitudes que le permitan participar en la sociedad y aprender a lo largo de la vida. Más concretamente, la European Commission (2007; 2018b) define las competencias clave para la ciudadanía argumentando que son necesarias para la ocupabilidad, el desarrollo personal, la ciudadanía activa y la inclusión social. La CD es una de las ocho competencias consideradas clave para un ciudadano, 
necesarias desde la primera infancia hasta el final de la vida adulta, que se desarrollan a lo largo de la vida en procesos de educación formal, no formal e informal:

"La CD implica el uso seguro, crítico y responsable, y el compromiso con las tecnologías digitales para el aprendizaje, en el trabajo y para la participación en la sociedad. Incluye alfabetización en información y datos, comunicación y colaboración, creación de contenido digital (incluida la programación), seguridad (incluido el bienestar digital y las competencias relacionadas con la ciberseguridad) y resolución de problemas" (European Commission, 2018b, p.4).

Compartimos la concepción sobre la CD de Ferrari, Punie y Redecker (2012) y Larraz (2013), entendida como la suma de diversas alfabetizaciones vinculadas a aspectos tecnológicos, informacionales, multimedia y comunicativos.

Se trata de una competencia compleja en cuyo desarrollo de la cual intervienen aspectos externos al ciudadano, además de la formación que pueda realizar cada persona a lo largo de su vida. Sánchez-Caballé, Gisbert y Esteve (2020) señalan que en la literatura científica se asocia el desarrollo de la CD a factores diversos relacionados con la edad (Prensky, 2001), situación socioeconómica, barreras lingüísticas, políticas educativas (Barlow-Jones y Westhuizen, 2011) o acceso a los dispositivos tecnológicos (Korucu et al., 2016).

La CD resulta imprescindible para aprender y participar en la sociedad, pero también resulta una competencia necesaria para poder acceder al trabajo. Desde la empleabilidad, la OECD (Falck, Heimisch y Wiederhold, 2016) y la UNESCO (2018) destacan la necesidad de disponer de CD para poder acceder a un complejo mercado laboral cada vez más digitalizado, sin duda un desafío para la educación del Siglo XXI. Esta competencia tiene un carácter transversal y nos permite mejorar otras competencias fundamentales para el empleo que están relacionadas con la comunicación, la colaboración, el trabajo en equipo, la resolución de problemas, la creatividad, entre otras (European Commission, 2012 y 2015).

La European Commision (2016) publicó el informe sobre las competencias digitales denominado DIGCOM: A Framework for Developing and Understanding Digital Competence in Europe, que pretende actuar como marco para el desarrollo y la comprensión de la competencia digital en Europa. Desde este proyecto se relaciona la competencia digital con el uso seguro, crítico y creativo de las TIC en relación al trabajo, la empleabilidad, el aprendizaje, el ocio, la inclusión y la participación en la sociedad (Ferrari, 2013). La propuesta considera 5 áreas: alfabetización en información y datos; comunicación y colaboración; creación de contenido; seguridad; resolución de problemas. Desde el proyecto DIGCOM se ha trabajado para la creación de un marco detallado de la competencia digital de todos los ciudadanos, a través de una descripción de las competencias que son necesarias para desenvolverse en la sociedad. DIGCOM busca identificar los componentes centrales que configuran las competencias digitales en términos de conocimiento, habilidades y actitudes necesarias para el logro dichas competencias, junto con desarrollar descriptores de competencia digital que sirvan para nutrir un modelo conceptual y directrices que puedan ser 
validadas a nivel europeo, considerando distintos modelos disponibles y, finalmente, proponer una carta de navegación para el posible uso y revisión de un modelo de competencias digitales (Vuorikari, Punie, Carretero y Van den Brande, 2016).

\section{BRECHA DIGITAL}

El término brecha digital describe una brecha en términos de acceso y uso de la tecnología de la información y la comunicación (Hibert, 2014). La desigualdad se plantea entre acceso a Internet y la calidad de la misma y el nivel de manejo de las competencias digitales.

El Plan Estratégico años 2018-2022 de la Comisión Interamericana de Telecomunicaciones (CITEL) busca promover en la ciudadanía la inclusión digital, reduciendo la brecha digital. Considera como meta, también, la de prestar atención a la población con mayor dificultad de acceso, entre ellas las personas mayores, para un mayor desarrollo de las competencias digitales (CITEL, 2018).

En la Cumbre Mundial sobre la Sociedad de la Información (CMSI, 2003), se acordó eliminar la brecha digital en el acceso a las tecnologías de la información y de la comunicación. En el año 2016, a nivel mundial, 3.500 millones de personas estaban utilizando Internet, de los cuales 2,5 millones eran de países en desarrollo (ITU, 2017). En Estados Unidos el 95\% de los adolescentes indican tener acceso teléfono inteligente, y el 45\% estar en línea (Anderson y Jiang, 2018). Entre 2005 y 2019, el número de los usuarios de Internet crecieron en promedio en un 10 por ciento todos los años (ITU, 2019). El 2019 la mitad de la población mundial tiene acceso a la Internet. El acceso a las tecnologías no ha contribuido al fin de las segregaciones que establece la sociedad del conocimiento; por el contrario, transfiere las categorías de inclusión y exclusión a otras dimensiones (Cobo, 2019).

La demanda de Internet en los hogares y el uso individual de las TD dependen no sólo del nivel de ingresos, sino también de otros factores sociodemográficos. Entre los más relevantes se encuentran la educación, el género, la ubicación geográfica (urbana o rural) y la presencia de niños en edad escolar en el hogar (Galperin, 2017).

Si abordamos la brecha debido a cuestiones de género, a nivel global, las mujeres tienen aproximadamente un $50 \%$ menos de probabilidades de estar conectadas que los hombres del mismo grupo de edad y con niveles de educación e ingresos familiares similares (Word Wide Web Foundation, 2015). Rubio y Escofet (2013) señalan que la experiencia de los hombres con las TIC es mayor que la de las mujeres, poseen antes ordenador, lo usan más, juegan más con él y lo utilizan más con finalidad de ocio, mientras que el uso de internet con fines educativos se da igual en hombres y mujeres. Rebollo-Catalán, Mayor-Buzon y García-Pérez (2017) realizaron una investigación en el uso de redes sociales por parte de mujeres, los resultados mostraron que la mayor parte de las mujeres encuestadas se perciben con un nivel medio-alto de competencias digitales en el uso de las redes sociales; y destacan especialmente en competencias emocionales, 
funcionales y de seguridad en las redes, mientras que presentan deficiencias en competencias informativas y creativas.

Existe una brecha digital de carácter generacional, entendida como las diferencias en cuanto al acceso y uso de las TIC en diferentes entornos sociales (Amaro, González y Matínez, 2020). La competencia digital, según Hernando y Phillippi (2013), no se adquiere por “inmersión tecnológica" y es necesario aprender a aprender; pero esta competencia es socialmente desigual y, entre otros aspectos, está ligada a la edad. En los países desarrollados, el 94\% de los jóvenes de 15 a 24 años usan Internet, comparado con el $67 \%$ en países en desarrollo y solo $30 \%$ en menos desarrollados (ITU, 2017).

La investigación de Amaro, González y Martínez (2020) evaluó las competencias digitales entre jóvenes universitarios y adultos mayores, y se encontraron enormes diferencias en torno a las diversas dimensiones de las competencias estudiadas entre ambas generaciones. En otra investigación que buscó medir el conocimiento y uso activo de una serie de ítems vinculados a la competencia digital por parte de 1.500 personas de distintas edades y niveles educativos, encontraron resultados que indican que el nivel del ciudadano medio no es el deseado, que existe una brecha digital generacional y de género, siendo el perfil medio más social, recreativo y consumidor de contenidos existentes, que proactivo, gestor y creador de contenidos propios (Dornaleteche-Ruiz, Buitrago-Alonso y Moreno-Cardenal, 2015).

\section{Medición o evaluación de la CD}

Junto al reto de la formación y el desarrollo de competencias, de forma paralela, coexiste el reto de evaluarlas o medirlas. El desarrollo de instrumentos de evaluación de la competencia digital que permitan automatizar el proceso y determinar el nivel de competencia del individuo resulta un tema relevante y presente en la literatura científica (González-Martínez, Esteve, Larraz, Espuny y Gisbert, 2018). La evaluación de esta competencia resulta útil, tanto desde una finalidad diagnóstica para orientar el proceso de enseñanza y aprendizaje, como desde una finalidad sumativa o incluso certificadora. Larraz, Espuny, Gisbert y Saz (2012), estudian diferentes herramientas de evaluación de la CD y concluyen que la mayoría están diseñadas paras el ámbito universitario y en pocas ocasiones consideran la totalidad de los componentes de la CD. Habitualmente se centran en alguno de ellos, siendo la alfabetización tecnológica el más recurrente.

Un ejemplo de evaluación certificadora de la CD para la ciudadanía es el que realiza la Generalitat de Catalunya mediante ACTIC (2020) (ACTIC - Acreditación de Competencias en Tecnologías de la Información y la Comunicación). Cualquier persona de más de 16 años puede realizar la prueba ACTIC, demostrar su CD y obtener un certificado que le permite acreditar el nivel de CD que, en muchas ocasiones, se considera un mérito e incluso un requisito para poder acceder a un trabajo en la administración pública o en la empresa privada. 
Una primera aproximación a la medición de las CD son los estudios de percepción de competencias digitales. En estudiantes universitarios muestran un nivel de manejo de CD sobrevalorado ya que los estudiantes perciben un manejo de TIC mayor al que realmente pueden demostrar. En el ámbito universitario, INCOTIC-grado es una herramienta diseñada para realizar una evaluación autodiagnóstica de la competencia digital de los estudiantes de primer curso de grado para poder ajustar la planificación de la docencia de esta competencia cuando se necesario (GonzálezMartínez, Espuny y Gisbert, 2011), generado en España, ahora con la versión INCOTIC-LA adaptado a Latinoamérica y que se encuentra en etapa piloto aplicandóse en universades Latiamericanas. Por su parte, ACUTIC ha sido elaborado para el estudio de las actitudes que tienen los estudiantes universitarios hacia las TIC (Mirete, García y Hernández, 2015).

Las pruebas estandarizadas no dan resultados concluyentes y emergen los enfoques que se orientan hacia las competencias del Siglo XXI o competencias TIC para los aprendizajes. Buscan trascender en el uso o habilidades digitales, y apuntan a un uso creativo y reflexivo para la resolución de problemas en un contexto de digitalización (Sunkel, Trucco y Espejo, 2013).

\section{Propuesta para desarrollar la CD}

El informe Eurydice (European Commission, 2019) pone de manifiesto que todos los países de la Unión Europea contemplan la CD en sus currículos, ya desde la educación primaria y secundaria obligatoria. De este modo se confirma la existencia de un enfoque estratégico de la educación digital en Europa, que puede constatarse mediante la inclusión de la CD como una competencia básica o clave en los diferentes sistemas educativos europeos. Según este informe, en la mayoría de países la $C D$ se concibe como una competencia transversal en los planes de estudio. Consideramos que este tipo de estrategia política en educación es imprescindible para poder formar en $C D$ a los futuros ciudadanos desde una edad temprana. El sistema educativo formal realiza una función niveladora y permite, de este modo, evitar algunos de los efectos de la brecha digital.

Considerando las aportaciones de diversas autores, Cabero-Almenara, Torres-Barzabal y Hermosilla-Rodríguez (2020) se plantean una serie de recomendaciones para la ciudadanía digital, hemos seleccionado y adaptado aquellas que serían útiles para el desarrollo de las competencias digitales en la ciudadanía:

a) a) Una adecuada formación en competencias digitales, que debe iniciarse en el marco de la educación formal (primaria, secundaria y educación superior).

b) b) continuar a lo largo de todos los procesos de formación permanente a lo largo de la vida. Y especialmente en contextos de educación no formal, sobre todo para sectores afectados por la brecha digital.

c) c) Favorecer e incentivar las prácticas innovadoras que faciliten la utilización colaborativa de las TD. 
d)

A partir de la realidad de conectividad en América Latina, Galperin (2017) propone recomendaciones para alimentar la política pública para una ciudadanía digital más inclusiva:

e) La conectividad de los establecimientos educativos en complementariedad con reformas curriculares y otras iniciativas de introducción de TIC en las escuelas.

f) El desarrollo de aplicaciones y contenido en línea orientados a atender las necesidades de grupos de baja conectividad, en particular adultos mayores, personas con discapacidad y hablantes de lenguas indígenas.

g) La inversión en capital humano a través de subsidios de conectividad focalizados en hogares de bajos ingresos con niños en edad escolar, condicionados en el cumplimiento de contraprestaciones educativas.

A este respecto, la Comisión Interamericana de Telecomunicaciones CITEL propone la producción y acceso de contenidos locales que les permita mayor apropiación de estas tecnologías; de tal forma que las tecnologías se transformen en facilitadoras del pleno desarrollo de las capacidades de la ciudadanía (CITEL, 2018).

Para Culver y Jacobson (2012) deben promoverse las competencias digitales en las personas mayores para su inclusión en la sociedad, mejorar su calidad de vida durante el proceso de envejecimiento, y ayudarles a poseer una vida social más activa y participativa. Esto implica proveerles de tecnología, capacitarlos y motivar sus usos, generar aplicaciones que respondan a las necesidades e inquietudes de este grupo etario. En países como Uruguay hay líneas de acción en esta dirección, al comprenderse las tecnologías como un fenómeno social.

Para Dornaleteche-Ruiz, Buitrago-Alonso y Moreno-Cardenal (2015), es necesario diseñar programas específicos para paliar la brecha de género, con el objetivo de idear propuestas para reducir la brecha digital entre generaciones, incidir en el empoderamiento de las mujeres a nivel tecnológico desde edades muy tempranas y en potenciar la vertiente expresiva, creativa y generadora de contenidos en Internet a través de cursos específicos diseñados para mujeres.

Algunas recomendaciones complementarias para el desarrollo de la competencia digital de la ciudadanía, surgidas a partir de la experiencia de la formación virtual en el contexto actual de COVID serían:

- Fomento del acceso a los espacios virtuales, aprovechando las potencialidades de las tecnologías digitales, plataformas virtuales y sus recursos, para favorecer el aprendizaje, el trabajo individual y colaborativo, integrarse a comunidades virtuales, conocer y utilizar las herramientas digitales que los gobiernos tienen para realizar trámites que antes se realizaban presenciales, conocer la importancia de evaluar la información para ver si es fidedigna, la importancia de la identidad de la presencia en esos espacios digitales. 
- Fomento de las habilidades y competencias digitales del siglo XXI, como elemento clave; trabajar con los estudiantes las habilidades de búsqueda de información, comunicación y colaboración, convivencia digital y habilidades tecnológicas. Además de las estrategias de aprendizaje autorreguladas, el autoaprendizaje, la metaevaluación y la metacognición.

- Fortalecimiento de las infraestructuras tecnológicas y acceso a la red. Es necesario disminuir la brecha digital del acceso a las tecnologías ampliando la cobertura, mejorando la calidad del servicio a precios accesibles por las personas. Este aspecto es relevante especialmente en los países en vías de desarrollo. En algunos casos será necesario contar con programas nacionales que garanticen este acceso y subvencionarlo si fuese necesario, disponibilizar de redes wifi de acceso gratuito.

- Diagnóstico de la CD y desarrollo de acciones formativas para la adquisición de las competencias digitales. Es necesario contar con un instrumento de evaluación del nivel de logro de las competencias digitales para, a la luz de los resultados, ofrecer a la ciudadanía experiencias formativas presenciales o virtuales. En este último caso deberían ser en modalidad e-learning, b-learning o MOOC. Esto será de vital importancia para desarrollar las competencias digitales e integrarlas a las acciones que normalmente desarrolla la ciudadanía en una sociedad altamente tecnologizada.

\section{CONCLUSIONES}

Para aprovechar las potencialidades de las tecnologías digitales, la sociedad actual exige un nivel competencia digital de la ciudadanía cada vez mayor. La emergencia sanitaria provocada por la pandemia del COVID19 ha puesto de manifiesto que esta competencia no la posee una gran parte de la ciudadanía. Las mayores dificultades están relacionadas con el uso de las tecnologías en el ámbito educativo, económico y laboral. En el ámbito personal y social se podría pensar que no existen tantas dificultades, pero aún se ponen de manifiesto que no se realiza un uso ético y seguro, pongamos por ejemplo todo aquello relacionado con las fake news.

Tanto en los países en vías de desarrollo como en los países desarrollados, la competencia digital se ve afectada por las desigualdades derivadas de la brecha digital, principalmente por el estatus socioeconómico, la raza, el género y la formación. Es necesario disminuir esta brecha de acceso y uso de la tecnología, pues consideramos que son aspectos fundamentales para avanzar en el desarrollo de una sociedad más equitativa y, por ende, más justa.

Contar con organismos que monitoreen el desarrollo de la competencia digital es prioritario en cada país. Y también se debería disponer de instrumentos de evaluación objetiva que permitan y 
midan el nivel de logro de la competencia digital. De este modo se podrán establecer mecanismos de apoyo precisos y políticas basadas en la evidencia de los resultados.

\section{REFERENCIAS}

ACTIC (2020). Acreditación de Competencias en Tecnologías de la Información y la Comunicación. Generalitat de Catalunya. https://bit.ly/2YsiP3a

Anderson, J. y Jiang, J., (2018) Teens-Social-Media-Technology 2018.http://publicservicesalliance.org/wp-content/uploads/2018/06/Teens-Social-MediaTechnology-2018-PEW.pdf

Amaro, A., González, E. y Martínez, N. (2020). Desafíos para una ciudadanía inclusiva: competencia digital entre adultos mayores y jóvenes, Comunicação Mídia e Consumo, 17(48), 11-33. http://dx.doi.org/10.18568/cmc.v17i48.2247

Barlow-Jones, G., y Westhuizen, D. (2011). Situating the student: Factors contributing to success in an Information Technology course. Educational Studies, 37 (3), 303-320. https://doi.org/10.1080/03055698.2010.506329

Cabezas, M., y Casillas, S. (2017). ¿Son los futuros educadores sociales residentes digitales? Revista Electrónica de Investigación Educativa, 19(4), 61-72. https://doi.org/10.24320/redie.2017.19.4.1369

Cabero-Almenara, J., Torres-Barzabal, L. y Hermosilla-Rodríguez, J. (2020). Las TIC y la creación de una ciudadanía crítica e-digital. Education in the Knowledge Society, 22, 1-20. https://doi.org/10.14201/eks2019 20 a22

CEPAL (2009). La sociedad de la información en América Latina y el Caribe. Santiago de Chile, cepal-idrc-Europe Aid. https://bit.ly/3alzmE8

CITEL -Comisión Interamericana de Telecomunicaciones (2018). RES. 79 (VII-18) Plan Estratégico 2018-2022. https://bit.ly/2C5H1AW

Cobo, C., y Moravec, J. (2011). Aprendizaje invisible: hacia una nueva ecología de la educación. Col·lecció Transmedia XXI. Laboratori de Mitjans Interactius. Publicacions i Edicions de la Universitat de Barcelona. Barcelona. https://www2.educationfutures.com/books/aprendizajeinvisible/es/

Cobo, Cristóbal (2019).Acepto las Condiciones: Usos y abusos de las tecnologías digitales, Fundación Santillana, Madrid. www.aceptolascondiciones.com 
Council of the EU (2017). Declaración de Roma. Retrieved from https://bit.ly/3cM

Culver, S.y Jacobson, T. (2012). Alfabetización mediática como método para fomentar la participación cívica. Comunicar, 39, 73-80. https://doi.org/10.3916/C39-2012-02-07

CMSI - Cumbre Mundial sobre la Sociedad de la Información (2003). Declaración de Principios: Construir la Sociedad de la Información: un desafío global para el nuevo milenio. https://bit.ly/3hmX3Xn

De la Selva, A. R. A. (2015). Los nuevos rostros de la desigualdad en el siglo XXI: la brecha digital. Revista mexicana de ciencias políticas y sociales, 60(223), 265-285. DOI: https://doi.org/10.1016/S0185-1918(15)72138-0

Duarte, F., y Pires, H. F. (2011). Inclusión digital, tres conceptos clave: conectividad, accesibilidad, comunicabilidad. Revista Electrónica de Recursos en Internet sobre Geografía y Ciencias Sociales, 150(1), 1-15. https://bit.ly/2TuxDwp

Dornaleteche-Ruiz, J., Buitrago-Alonso, A. y Moreno-Cardenal, L. (2015). Categorización, selección de ítems y aplicación del test de alfabetización digital online como indicador de la competencia mediática, Comunicar, 44, 177-185. http://dx.doi.org/10.3916/C44-2015$\underline{19}$

EUCO (2017). Conclusiones del Consejo Europeo de 19 de octubre de 2017. https://bit.ly/2z7w8gV

European Commission (2007). Key Competencies for Lifelong Learning: European Reference Framework, Office for Official Publications of the European Communities, Luxembourg. https://bit.ly/3h20QZL

European Commission (2012). ESCO (clasificación europea de capacidades/competencias, cualificaciones y ocupaciones). https://bit.ly/2MLnJmy

European Commission (2015). Marco estratégico: Educación y formación 2020. https://bit.ly/37hKR5C

European Commission. (2016). DIGCOMP 2.0: The digital competence framework for citizens. Recuperado de https://goo.gl/efttR1

European Commission (2017). European Skills, Competences, Qualifications and Occupations. https://bit.ly/3cW5IgE

European Commission (2018a). Comunicación de la comisión al parlamento europeo, al consejo, al comité económico y social europeo y al comité de las regiones sobre el Plan de Acción de Educación Digital. https://bit.ly/3e4ZPhm 
European Commission (2018b). Proposal for a council recommendation on key competences for lifelong learning. https://bit.ly/3cKjBO5

European Commission (2019). Digital Education at School in Europe. Eurydice Report. Luxembourg: Publications Office of the European Union. https://bit.ly/2Yhe1XA

Falck, O., Heimisch, A. y Wiederhold, S. (2016), "Returns to ICT Skills", OECD Education Working Papers, No. 134, OECD Publishing, Paris. http://dx.doi.org/10.1787/5jlzfl2p5rzq-en

Fernández, C. (2017). Un desafío en la inclusión ciudadana. Telos: Fundación Telefónica, 107. https://bit.ly/2KEHVp9

Ferrari, A., Punie, Y. y Redecker, C. (2012). Understanding digital competence in the 21st century: An analysis of current frameworks. In European Conference on Technology Enhanced Learning (pp. 79-92). Springer, Berlin, Heidelberg. http://dx.doi.org/10.1007/978-3-64233263-0 7

Ferrari, A. (2013). DIGCOMP: A framework for developing and understanding digital competence in Europe. https://bit.ly/2BZvjaQ

Fundación Telefónica (2020). Sociedad digital en España 2019. https://bit.ly/2ZOnpul

Galperin, H (2017). Sociedad digital: brechas y retos para la inclusión digital en América Latina y el Caribe, Montevideo:Unesco. https://bit.ly/2Uipw6D

Gisbert, M. y Esteve, F. (2011). Digital learners: la competencia digital de los estudiantes universitarios. La Cuestión Universitaria, 7. https://bit.ly/3gANTWP

González-Martínez, J., Espuny, C., y Gisbert, M. (2011). INCOTIC. Una herramienta para la autoevaluación diagnóstica de la competencia digital en la universidad. Profesorado: Revista currículum y formación del profesorado. 15(1), 75-90.

González-Martínez, J., Esteve, F. M., Larraz, V., Espuny, C., y Gisbert, M. (2018). INCOTIC 2.0: una nueva herramienta para la autoevaluación de la competencia digital del alumnado universitario. Profesorado: Revista de currículum y formación del profesorado, 22(4), 133152. Doi:10.30827/profesorado.v22i4.8401

Hernado, A. y Phillippi, A. (2013). El desarrollo de la competencia mediática en personas mayores: una brecha pendiente. Chasqui, 124,12-18.

Hilbert, Martin (2014). Technological information inequality as an incessantly moving target: The redistribution of information and communication capacities between 1986 and 2010. 
Journal of the Association for Information Science and Technology, 65 (4), 821-835 http://dx.doi.org/10.1002/asi.23020

ITU (2017). ICT facts and figures. https://bit.ly/2UK3ha1

ITU (2019). Measuring digital development Facts and figures. https://bit.ly/3dVXndn

Korucu, A. T., Yucel, A., Gundogdu, M. M., y Gencturk, T. (2016). Investigation the Technology Usage Level of Teacher Candidates. Participatory Educational Research, 3(1), 14-21. https://doi.org/10.17275/per.15.49.3.1

Larraz, V., Espuny, C., Gisbert, M. y Saz A. (2012). Las herramientas para la evaluación de la competencia digital. Análisis y componentes. EDUTEC. Gran Canaria, 2012.

Larraz, V. (2013). La competència digital a la universitat. Tesi doctoral. Universitat d’Andorra. Identificador: TD-017-100006/201210.

Lázaro Cantabrana, J. L., Estebanell Minguell, M., y Tedesco, J. C. (2015). Inclusion and Social Cohesion in a Digital Society. RUSC. Universities and Knowledge Society Journal, 12(2). pp. 44-58. http://dx.doi.org/10.7238/rusc.v12i2.2459

Mirete, A., García, F. y Hernández, F. (2015).Cuestionario para el estudio de la actitud, el conocimiento y el uso de TIC (ACUTIC) en Educación Superior. Estudio de fiabilidad y validez. Revista Interuniversitaria de Formación del Profesorado, 83 (29.2),75-89.

Prensky, M. (2001). Digital natives, digital immigrants. On the Horizon, 9(5), 1-6.

Rebollo-Catalán, A., Mayor-Buzon, V. y García-Pérez, R. (2017). Competencias digitales de las mujeres en el uso de las redes sociales virtuales: diferencias según perfil laboral. Revista de Investigación Educativa, 35(2), 427-444 http://dx.doi.org/10.6018/rie.35.2.270881

Rubio, M.J., y Escofet, A. (2013). Estudio sobre los usos de las TIC y las posibilidades de empoderamiento en las mujeres. Revista Iberoamericana de Educación, 62 (3), 1-13.

Sánchez-Caballé, A., Gisbert, M., y Esteve, F. (2020). The digital competence of university students: a systematic literature review. Aloma: Revista de Psicologia, Ciències de I'Educació i de l'Esport, 38(1). https://bit.ly/2XK6nvr

Sunkel, G., Trucco, D. y Espejo, A. (2013) La integración de las tecnologías digitales en las escuelas de América Latina y el Caribe. Una mirada multidimensional. Santiago: Comisión Económica para América Latina y el Caribe (CEPAL). 
Tello, E. (2007). Las tecnologías de la información y comunicaciones (TIC) y la brecha digital: su impacto en la sociedad de México. International Journal of Educational Technology in Higher Education (ETHE). http://dx.doi.org/10.7238/rusc.v4i2.305

United Nations (2015). Transforming our world:the 2030 agenda for sustainable development. https://bit.ly/2yKKRhc

Unesco (2018). ICT Competency Framework for Teachers. https://bit.ly/34IRJx3

Vuorikari, R., Punie, Y., Carretero Gomez S. y Van den Brande, G. (2016). DigComp 2.0: The Digital Competence Framework for Citizens. Update Phase 1: The Conceptual Reference Model. Luxembourg Publication Office of the European Union. http://dx.doi.org/10.2791/11517

Word Wide Web Foundation (2015) Women's Rights Online Translating Access into Empowerment. https://bit.ly/2XYLwWe

Para citar este artículo:

Silva Quiroz, J. E., y Lázaro-Cantabrana, J. L. (2020). La competencia digital de la ciudadanía, una necesidad creciente en una sociedad digitalizada. Edutec. Revista Electrónica De Tecnología Educativa, (73), 37-50. https://doi.org/10.21556/edutec.2020.73.1743 